\title{
The Impact of Extensive Cytoreductive Surgery for Ovarian Cancer on the Histopathology Laboratory Workload
}

\section{Ahmed El-Masry ${ }^{* 1}$ and Mona El-Bahrawy ${ }^{2,3}$}

${ }^{1}$ Faculty of Medicine, Department of Histopathology, Imperial College London, UK

${ }^{2}$ Department of Histopathology, Imperial College London, UK

${ }^{3}$ Department of Pathology, Alexandria University, Egypt

\begin{abstract}
Objectives: Cytoreductive surgery is the principal management for ovarian cancer. Recently there has been progressive change to more extensive cytoreductive surgery (ECS) as evidence shows this improves patient prognosis. The aim of this study is to investigate the change in histopathology work load with change in surgical practice for the treatment of ovarian cancer patients at Hammersmith Hospital, UK.

Materials and methods: Specimens for patients with ovarian cancer $(n=116)$ were selected and classified into three groups: (i) standard debulking surgery; (ii) a mix of standard debulking and ECS and (iii) ECS only. The types of specimens and numbers of blocks in each group were studied.

Results: Post-hoc analysis demonstrates a statistically significant increase in the number of specimens per case from standard debulking to the mixed group $(p<0.0001)$ and to the ECS group $(p<0.0001)$. There is also a statistically significant increase in the number of blocks from standard debulking to the mixed $(p<0.0001)$ and to the ECS groups $(p<0.0001)$.

Conclusion: The study shows there is a significant increase in the histopathology workload with the shift from standard to extensive cytoreductive surgery, as well as increase in the complexity and range of specimens sent for histopathological examination. It is essential that centres opting for a shift to ECS ensure that adequate provisions and resources are in place to accommodate these changes.
\end{abstract}

Keywords: Ovarian; Cancer; Debulking; Workload

Abbreviations: ECS: Extensive Cytoreductive Surgery; NICE: National Institute for Health and Clinical Excellence; NGR: No Gross Residual Disease; OS: Overall Survival; RD: Residual Disease

\section{Introduction}

Ovarian cancer is the sixth commonest cancer among women worldwide and is the leading cause of cancer related deaths in women with gynaecological malignancies [1]. With an increasing burden of disease and cases typically presenting with advanced-stage disease, managing these patients is complex, requiring both optimal planning and a multi-disciplinary approach to treatment.

For decades, cytoreductive surgical management has been the mainstay of therapy for advanced-stage ovarian cancer. Primary cytoreduction is often followed by platinum or taxane based combination chemotherapy in more disseminated disease [2,3]. The landmark study by Griffiths in 1975 demonstrated the survival benefit of maximal tumour debulking [4]. Following this seminal paper, overwhelming evidence from multiple institutional retrospective series and pooled data from prospective randomized chemotherapy trials have confirmed the role of cytoreductive surgery in prolonging survival [5-9]. The efficacy of surgery was measured in regards to achieving optimal cytoreduction, with patient survival demonstrated to be inversely correlated with postoperative residual tumour burden $[4,8,10-12]$.

Studies have shown that residual disease (RD) after initial surgery is a strong independent prognostic factor for survival and recurrence, with improvements in both overall and progression free survival being greatest in women with no (currently termed complete cytoreduction) or minimal $(<1 \mathrm{~cm}$, currently termed optimal cytoreduction) visible residual disease post-operatively [4-6,12-14]. A number of studies have consistently shown that optimal cytoreduction is associated with a significant survival benefit in patients with this disease $[8,11,14]$.
However, several recent studies have focused on the survival benefit associated with no gross residual disease (NGR) achieved from maximal surgical resection and demonstrated that patients with NGR after primary cytoreduction have significantly longer median overall survival (OS) time than those with any $\mathrm{RD}[5,6,9,12,15]$. Standard surgery is defined as total abdominal hysterectomy, bilateral salpingo-oophorectomy, omentectomy either with or without removal of enlarged lymph nodes (para-aortic, pelvic and obturator), and debulking of any other superficial tumour plaques [5,16]. Radical surgery comprises, in addition to the above mentioned elements, en bloc removal of the uterus, both ovaries, pelvic peritoneum and rectosigmoid, with or without simple peritonectomies.

In advanced stage ovarian cancer, tumour spread across the abdomen may involve, in different combinations, the diaphragm, the spleen, distal pancreas, the lesser sac, the liver, the gallbladder, the porta hepatis, the stomach, and the peritoneal surfaces surrounding all these areas. In these cases, upper abdominal disease is associated with worse prognosis when compared to patients with disease limited to the pelvis [17].

For a significant period of time, tumour spreading to the upper abdomen was considered non-resectable disease and thus was an

*Corresponding author: El-Masry ANS, C/O Dr Mona El-Bahrawy, Department of Histopathology, Hammersmith Hospital, DuCane Road, London, W12 0NN, UK Tel: 0044 (0)208 383 3442; E-mail: ahmed.el-masry12@imperial.ac.uk

Received April 27, 2017; Accepted June 20, 2017; Published June 28, 2017

Citation: El-Masry A, El-Bahrawy M (2017) The Impact of Extensive Cytoreductive Surgery for Ovarian Cancer on the Histopathology Laboratory Workload. J Cytol Histol 8: 454. doi: 10.4172/2157-7099.1000454

Copyright: (C) 2017 El-Masry A, et al. This is an open-access article distributed under the terms of the Creative Commons Attribution License, which permits unrestricted use, distribution, and reproduction in any medium, provided the original author and source are credited. 
indication for neo-adjuvant chemotherapy or palliation. However, with improved surgical expertise in the management of ovarian cancer and a shift in surgical training to a more radical approach to tumour debulking, came the introduction of extensive cytoreductive surgery (ECS), described in the literature as treatment for advancedstage disease with low complication rates, acceptable morbidity and equivalent outcomes [18-21].

In accordance to the National Institute for Health and Clinical Excellence (NICE) guidance, ECS can be defined as a further development to radical surgical management and typically features, "stripping of the diaphragm, extensive stripping of the peritoneum, multiple resections of the bowel (excluding localised colonic resection), liver resection, partial gastrectomy, cholecystectomy, and splenectomy" [22].

In many centres, upper abdominal procedures are now performed for surgical cytoreduction including diaphragmatic peritonectomy or full thickness resection, splenectomy with or without distal pancreatectomy, cholecystectomy and resection of parenchymal liver disease and porta hepatis disease [23-25]. In addition to this, surgeons have also introduced transdiaphragmatic resection of pleural and mediastinal disease during an abdominal debulking procedure [26].

Current surgical practice has demonstrated that, in appropriately selected patients, most of these disease locations are amenable to cytoreduction with an associated improvement in patient outcomes, and with manageable patient morbidity $[27,28]$.

As would be expected, ECS is associated with prolonged operating time and increased exposure to anaesthesia. In addition, these women may require a longer hospital stay and recovery time, with an increased risk of complications. The impact of all these factors on surgical departments and wards involved in the care of these patients as well as patient outcomes (survival, complication and recurrence rates) are continuously explored, with official protocols likely to be published in the coming years.

With its likely implementation on a large scale, the cost of ECS requires evaluation. One of the costs that should be considered, often being overlooked, is the cost and resources required for histopathological evaluation of all the resected specimens. Receipt of the specimens in the histopathology laboratory starts a procedure of multiple steps, requiring the input of medical, technical and administrative staff, all of which must be evaluated [29].

The practice at the Hammersmith Hospital for the management of advanced ovarian cancer patients has progressively changed towards ECS over the past few years. The aim of this study was to assess the change in the histopathology work load with the progressive adoption of ECS.

\section{Materials and Methods}

This is a single centre retrospective analysis of the histopathological evaluation work for patients who underwent cytoreductive surgery for advanced primary or relapsed ovarian cancer at Hammersmith Hospital, Imperial College Healthcare NHS Trust, UK. This study was approved by the Imperial College NHS Trust Tissue Bank Institutional Review Board (R10008).

Information extracted from the histopathology database included the number of specimens and blocks as well as tissue type/origin making up the histopathologist workload when investigating samples from these patients, who were admitted between January 2005 and December 2015; as surgical management shifted to a more extensive approach.

\section{Study design}

This study includes 116 cases of patients who underwent surgery for ovarian carcinoma, FIGO stage III or IV. The cases were classified into 3 groups: (i) standard debulking surgery; (ii) a mix of standard debulking and ECS and (iii) ECS alone.

The composition of these study groups allows for the assessment of histopathologist workload over the evolution of surgical management practice for advanced ovarian cancer. The standard debulking group $(n=50)$, demonstrates the work load prior to recent changes in surgical practice. The mixed group reflects current practice following the introduction of ECS. These patients $(n=50)$ were gathered consecutively and demonstrate the average cohort of patients currently managed within the hospital, with a mix of standard $(n=16)$ and extensive $(n=34)$ surgery employed. The lone ECS group reflects the expected workload where ECS is the only adopted surgical approach. Although originally containing 16 patients, the 34 patients from the mixed group who underwent ECS were also added as data sets to the ECS group. This gives us 50 patients assessed within each of the three groups to facilitate statistical analysis (Figure 1).

In this study, ECS is defined in accordance to NICE guidelines, [22] with patient inclusion in this group determined by having a surgical complexity score (SCS) $\geq 5$; calculated using the Mayo Clinic Surgical Complexity Score (SCS) scoring system [30].

\section{Statistical analysis}

To statistically assess differences in workload between groups, nonparametric Kruskal-Wallis $\mathrm{H}$ test was employed. Analyses were performed using the GraphPad Prism 7.0 software package (San Diego, CA) with a significance threshold of $p \leq 0.05$.

\section{Results}

\section{Patient characteristics}

Patients' age ranged between $30-86$ years. All patients had ovarian high grade serous carcinoma, FIGO stage III or IV. Details are presented in Table 1.

\section{Numbers of specimens and blocks sent for examination}

In the standard surgery group the number of specimens submitted per case ranges from 1-7 specimens (Median, 3 and mean, 2.72) and the number of blocks per case ranges from 7-46 (Median, 23 and mean, 24.1).

In the mixed group the number of specimens submitted per case ranges from 2-20 specimens (Median, 10 and mean, 9.7) and the number of blocks per case ranges from 23-103 specimens (Median, 49 and mean 57.3).

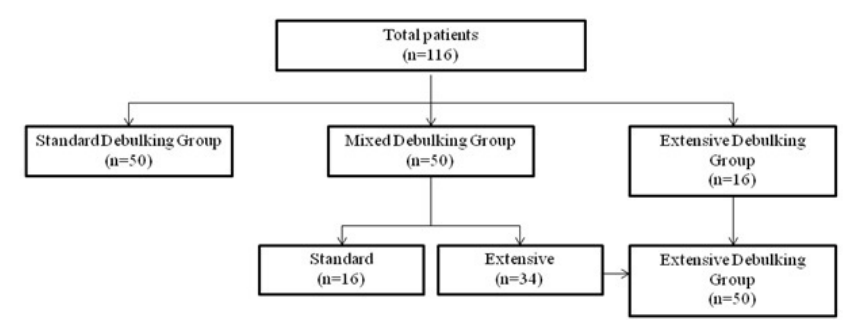

Figure 1: Groups of patients studied ( $n=116)$ : (i) standard debulking surgery; (ii) a mix of standard debulking and extensive surgery and (iii) extensive surgery alone; reflecting the evolution of practice. 
In the ECS group the number of specimens submitted per case ranges from 8-21 specimens (Median, 14 and mean 12) and the number of blocks per case ranges from 22-135 specimens (Median, 69 and mean 67.2).

The differences in specimen and block counts across surgical practice groups is demonstrated in Figure 2. There is a statistically significant difference in both specimen $(\mathrm{H}=90.2, \mathrm{p}<0.0001)$ and block $(\mathrm{H}=82$, $\mathrm{p}<0.0001)$ counts across all groups. There is a 3.5 fold increase in mean specimen number from the standard debulking to the mixed group and 4.4 fold increases from the standard to the ECS group, and 2.4 fold increase in mean block number from the standard debulking to the mixed group and 2.8 fold from the standard to the ECS group.

Post-hoc analysis demonstrates a statistically significant increase in the number of specimens per case from standard debulking to the mixed group $(\mathrm{p}<0.0001)$ and to the ECS group $(\mathrm{p}<0.0001)$, with a nonsignificant difference between the mixed and ECS group $(\mathrm{p}=0.08)$; with means of 2.72, 9.7 and 12 respectively.

There is also a statistically significant increase in the number of blocks from standard debulking to the mixed group $(\mathrm{p}<0.0001)$ and to the ECS group $(\mathrm{p}<0.0001)$, with a non-significant difference between the mixed debulking and ECS group $(\mathrm{p}=0.19)$; with means of $24.1,57.3$ and 67.2 respectively.

\section{Tissue type and origin of resected specimens}

With regards to the tissue type and origin of specimens sent to the laboratory for examination, a greater variety of specimens and case complexity is observed. Table 2 displays the different specimens examined, with the number of times each was encountered in the included cases.

There are types of specimens only encountered in cases of ECS, including spleen, liver tissue, pancreatic tissue, peritoneal and diaphragmatic tumour deposits, mesenteric tumour deposits, tumour deposits on the surface of visceral organs such as the liver and kidney, bladder resections and ovarian vessels.

A significant increase in the frequency and extent of lymph node dissection is observed with groups that are not encountered in the standard debulking surgery and only included in the ECS group such as para-aortic, pelvic, renal, obturator, abdominal, paracaval and inguinal lymph nodes.

\section{Discussion}

The cornerstone of management of ovarian cancer is the combination of maximal surgical effort and chemotherapy. Both components require a high level of expertise and can only be provided in centres with the appropriate surgical, medical, and nursing resources. Resources and expertise for histopathological examination should also be secured for appropriate handling and reporting on the resected material.

The results of this study demonstrate that a significant change in the pathologists' workload is to be expected with the introduction of ECS. In addition to the significant increase in specimen and block numbers the introduction of multi-organ resection in the ECS group represents a significant change in complexity of specimens in these cases.

The dissection of lymph nodes is also an integral part of ECS including multiple lymph node groups. This also represents a significant change as lymph node dissection specimens require meticulous examination to harvest all included lymph nodes on macroscopic examination and microscopic examination of lymph nodes for tumour metastasis requires much time to be done with the due diligence.

It is important to match case volume and complexity growth to the staffing levels for pathology laboratories, including medical and technical staff [31]. Greater test complexity requires higher levels of professional medical, scientific and technical input in terms of time and expertise [32]. Since staff costs account for the largest single proportion

\begin{tabular}{|c|c|c|c|}
\hline & Standard Debulking & Mixed Debulking & Extensive Debulking \\
\hline Age Median (Range) & $65(30-81)$ & $67(32-84)$ & $64(32-86)$ \\
\hline \multicolumn{4}{|c|}{ Histology } \\
\hline High Grade Serous Carcinoma & 50 & 50 & 50 \\
\hline \multicolumn{4}{|c|}{ FIGO Stage } \\
\hline III & 43 & 44 & 40 \\
\hline IV & 7 & 6 & 10 \\
\hline
\end{tabular}

Table 1: Patient and tumour characteristics $(n=116)$.

A Variation in number of parts with change in surgical practice

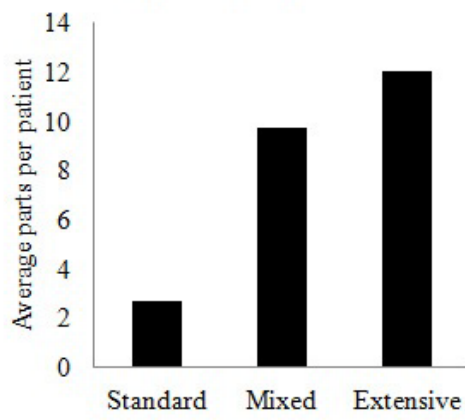
Variation in number of blocks with change in surgical practice

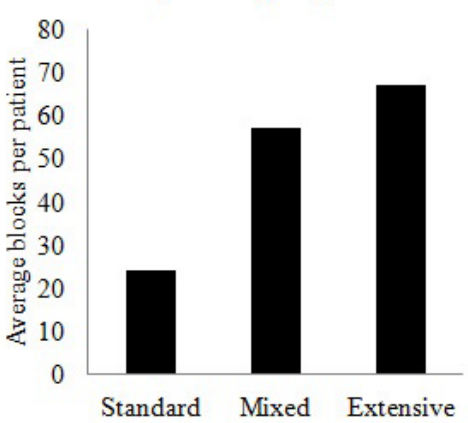

Figure 2: Effect of evolution in surgical practice for ovarian tumour debulking on (A) mean number of specimens sent for examination; (B) mean number of blocks selected per case. 


\begin{tabular}{|c|c|c|}
\hline & Standard Debulking & Extensive Debulking \\
\hline Uterus, fallopian tubes and ovaries & 44 & 45 \\
\hline Ovaries and fallopian tubes & 25 & 3 \\
\hline Ovarian vessel & & 26 \\
\hline Bowel & 13 & 66 \\
\hline Donuts & 4 & 19 \\
\hline Appendix & 4 & 29 \\
\hline Mesenteric deposit & & 51 \\
\hline Liver tissue & & 3 \\
\hline Liver capsule & & 14 \\
\hline Gall bladder & & 2 \\
\hline Pancreas & & 1 \\
\hline Renal capsule & & 1 \\
\hline Ureter & 1 & 2 \\
\hline Bladder & & 2 \\
\hline Peritoneal deposit & 3 & 67 \\
\hline Pouch of Douglas & 4 & \\
\hline Morrison Pouch & & 10 \\
\hline Ligamentum Teres & & 1 \\
\hline Falciform Ligament & & 26 \\
\hline Diaphragmatic deposits & & 25 \\
\hline Abdominal wall & 2 & 8 \\
\hline Port site metastases & 1 & 1 \\
\hline Umbilicus & & 5 \\
\hline Common Iliac In & 1 & 6 \\
\hline Internal Iliac In & 3 & 2 \\
\hline External Iliac In & 1 & 8 \\
\hline Mesenteric In & 1 & 5 \\
\hline Para aortic In & & 28 \\
\hline Pelvic In & & 36 \\
\hline Renal In & & 16 \\
\hline Abdominal In & & 18 \\
\hline Obturator In & & 8 \\
\hline Paracaval In & & 11 \\
\hline Inguinal In & & 1 \\
\hline Non-specified (NOS) In & 2 & 1 \\
\hline Spleen & & 15 \\
\hline Omentum & 38 & 54 \\
\hline Pelvic mass & 2 & 3 \\
\hline
\end{tabular}

Table 2: Resected specimen types and number of times encountered in the standard surgery $(n=50)$ and ECS $(n=50)$ groups.

of laboratory costs, test complexity has a major impact on costs, and so test complexity and test mix needs to be taken into consideration when undertaking cost estimation [32]. Service quality considerations, including efficiency and effectiveness should also be taken into account [33].

Service provision in anatomic pathology laboratories includes all facets of the technical work, leading up to pathologist's review and the rendering of a diagnosis [34]. Receipt of the specimens in the histopathology laboratory starts a procedure of multiple steps. These steps start with accessioning, which is the receipt and verification of specimens and their related requisitions for testing procedures, the subsequent order entry into the laboratory information system, and the assignment of a unique identifier that is associated with all related specimen materials [29]. This is followed by gross examination of the surgical specimens and the related order requisition to include specimen identification, clinical history, specimen description, number and location of blocks generated, with each block uniquely identified [29]. Grossing and block selection is a principal step in specimen assessment, one that is time consuming when handling complex specimens [35]. The tissue blocks then undergo processing to prepare stained slides for microscopic histopathological evaluation by the histopathologists [29].

Throughout all procedures, constant verification between specimen, paperwork, blocks, and slides is needed, which is an essential quality assurance activity for which all laboratory personnel are responsible [29]. This requires optimal staffing levels within the anatomic pathology laboratories, including the laboratory assistants, laboratory technicians, pathologists and administrators, all working within an increasingly complex regulatory environment [29].

All specimens submitted to the department are processed and pathologists are expected to read, interpret, and report all specimens assigned to them. So it is essential that as the workload rises, so should the number of pathologist and laboratory staff full-time equivalents to perform duties adequately [36].

On transition from the practice of standard surgery to ECS there may not be increase in the number of cases submitted to a Histopathology Department, but there is significant increase in the amount of material submitted for assessment per case. Hence in this study we reviewed the number of specimens submitted and the number of blocks selected for each case. We found that the mean number of specimens submitted rises up to 4 folds and the number of blocks per case rises over 2 folds on transition from standard surgery to ECS. Simple measurement of case numbers fails to adequately represent the work of a laboratory. Numbers of blocks and hence of slides reviewed and total lines of factual data provided are strong correlates of the time required to complete case review, report construction and verification [37]. Guidelines provided by the Royal College of Pathologists aim to provide a mechanism for assessing an individual's workload [38]. However, these still do not take into account many changes in requirements progressively introduced that increases the workload of pathologists. The complexity of the cases reviewed results in significant variance in the effort and time required to bring a case to final diagnosis.

\section{Conclusion}

Our study shows significant change in work load occurs even with partial introduction of ECS. The results show a significant difference between the group undergoing standard surgery and the groups undergoing ESC in whole or in part ( $<<0.0001$ across the board), while no significant difference is found between groups representing mixed cases and exclusively ECS ( $\mathrm{p}=0.08$ (specimens) and $\mathrm{p}=0.19$ (blocks)). Thus, a complete shift from standard to extensive surgical practice does not need to occur before histopathological workload is impacted. Therefore, it is essential for individual laboratories to implement safeguards prior to introducing ECS within their hospital or trust. Adequate resources, staffing and expertise must be implemented prior to considering any degree of change in surgical practice to maintain a high standard of service delivery.

Our results demonstrate the extent of impact of this change in practice that may help laboratories in addressing staffing requirements and resource allocation when planning to adopt this change in practice in their institutions.

\section{References}

1. Coleman MP, Forman D, Bryant H, Butler J, Rachet B, et al. (2011) Cancer survival in Australia, Canada, Denmark, Norway, Sweden, and the UK, 1995 2007 (the International Cancer Benchmarking Partnership): an analysis of population-based cancer registry data. Lancet 377: 127-138. 
Citation: El-Masry A, El-Bahrawy M (2017) The Impact of Extensive Cytoreductive Surgery for Ovarian Cancer on the Histopathology Laboratory Workload. J Cytol Histol 8: 454. doi: 10.4172/2157-7099.1000454

2. Chang SJ, Bristow RE (2012) Evolution of surgical treatment paradigms for advanced-stage ovarian cancer: redefining 'optimal' residual disease. Gynecologic oncology 125: 483-492.

3. Ozols RF, Bundy BN, Greer BE, Fowler JM, Clarke-Pearson D, et al. (2003) Phase III trial of carboplatin and paclitaxel compared with cisplatin and paclitaxel in patients with optimally resected stage III ovarian cancer: a Gynecologic Oncology Group study. Journal of clinical oncology 21: 3194-3200.

4. Griffiths CT (1975) Surgical resection of tumor bulk in the primary treatment of ovarian carcinoma. National Cancer Institute monograph 42: 101-104.

5. Aletti GD, Dowdy SC, Gostout BS, Jones MB, Stanhope CR, et al. (2006) Aggressive surgical effort and improved survival in advanced-stage ovarian cancer. Obstetrics and Gynecology 107: 77-85

6. du Bois A, Reuss A, Pujade-Lauraine E, Harter P, Ray-Coquard I, et al. (2009) Role of surgical outcome as prognostic factor in advanced epithelial ovarian cancer: a combined exploratory analysis of 3 prospectively randomized phase 3 multicenter trials: by the Arbeitsgemeinschaft Gynaekologische Onkologie Studiengruppe Ovarialkarzinom (AGO-OVAR) and the Groupe d'Investigateurs Nationaux Pour les Etudes des Cancers de l'Ovaire (GINECO). Cancer 115: 1234-1244.

7. Eisenkop SM, Spirtos NM (2001) Procedures required to accomplish complete cytoreduction of ovarian cancer: is there a correlation with "biological aggressiveness" and survival? Gynecologic oncology 82: 435-441.

8. Hoskins WJ, McGuire WP, Brady MF, Homesley HD, Creasman WT, et al (1994) The effect of diameter of largest residual disease on survival after primary cytoreductive surgery in patients with suboptimal residual epithelial ovarian carcinoma. American journal of obstetrics and gynecology 170: 974979

9. Winter WE 3rd, Maxwell GL, Tian C, Carlson JW, Ozols RF, et al. (2007) Prognostic factors for stage III epithelial ovarian cancer: a Gynecologic Oncology Group Study. Journal of clinical oncology 25: 3621-3627.

10. Bristow RE, Montz FJ, Lagasse LD, Leuchter RS, Karlan BY (1999) Survival impact of surgical cytoreduction in stage IV epithelial ovarian cancer Gynecologic oncology 72: 278-287.

11. Bristow RE, Tomacruz RS, Armstrong DK, Trimble EL, Montz FJ (2002) Surviva effect of maximal cytoreductive surgery for advanced ovarian carcinoma during the platinum era: a meta-analysis. Journal of clinical oncology 20: 1248-1259.

12. Eisenkop SM, Friedman RL, Wang HJ (1998) Complete cytoreductive surgery is feasible and maximizes survival in patients with advanced epithelial ovarian cancer: a prospective study. Gynecologic oncology 69: 103-108

13. Omura GA, Brady MF, Homesley HD, Yordan E, Major FJ, et al. (1991) Longterm follow-up and prognostic factor analysis in advanced ovarian carcinoma: the Gynecologic Oncology Group experience. Journal of clinical oncology 9: 1138-1150.

14. Shih KK, Chi DS (2010) Maximal cytoreductive effort in epithelial ovarian cancer surgery. Journal of gynecologic oncology 21: 75-80.

15. Winter WE 3rd, Maxwell GL, Tian C, Sundborg MJ, Rose GS, et al. (2008) Tumor residual after surgical cytoreduction in prediction of clinical outcome in stage IV epithelial ovarian cancer: a Gynecologic Oncology Group Study. Journal of clinical oncology 26: 83-89.

16. Todo Y, Sakuragi N, Oikawa M, Negishi H, Yamamoto R, et al. (2003) Cytoreductive surgery combined with organ resection for advanced ovarian carcinoma. International journal of clinical oncology 8: 90-96.

17. Hamilton CA, Miller A, Miller C, Krivak TC, Farley JH, et al. (2011) The impact of disease distribution on survival in patients with stage III epithelial ovarian cancer cytoreduced to microscopic residual: a Gynecologic Oncology Group study. Gynecologic oncology 122: 521-526.

18. Bristow RE, del Carmen MG, Kaufman HS, Montz FJ. (2003) Radical oophorectomy with primary stapled colorectal anastomosis for resection of locally advanced epithelial ovarian cancer. Journal of the American College of Surgeons 197: 565-574.

19. Jaeger W, Ackermann S, Kessler H, Katalinic A, Lang N, et al. (2001) The effect of bowel resection on survival in advanced epithelial ovarian cancer. Gynecologic oncology 83: 286-291.

20. Merideth MA, Cliby WA, Keeney GL, Lesnick TG, Nagorney DM, et al. (2003) Hepatic resection for metachronous metastases from ovarian carcinoma Gynecologic oncology 89: 16-21.
21. Montz FJ, Schlaerth JB, Berek JS (1989) Resection of diaphragmatic peritoneum and muscle: role in cytoreductive surgery for ovarian cancer. Gynecologic oncology 35: 338-40.

22. NIfCE (2013) Ultra-radical (extensive) surgery for advanced ovarian cancer. Interventional procedures guidance In: NICE, ed. Interventional procedures guidance [IPG470].

23. Cliby W, Dowdy S, Feitoza SS, Gostout BS, Podratz KC (2004) Diaphragm resection for ovarian cancer: technique and short-term complications. Gynecologic oncology 94: 655-660.

24. Dowdy SC, Loewen RT, Aletti G, Feitoza SS, Cliby W (2008) Assessment of outcomes and morbidity following diaphragmatic peritonectomy for women with ovarian carcinoma. Gynecologic oncology 109: 303-307.

25. Magtibay PM, Adams PB, Silverman MB, Cha SS, Podratz KC, et al. (2006) Splenectomy as part of cytoreductive surgery in ovarian cancer. Gynecologic oncology 102: 369-374.

26. Diaz JP, Abu-Rustum NR, Sonoda Y, Downey RJ, Park BJ, et al. (2010) Videoassisted thoracic surgery (VATS) evaluation of pleural effusions in patients with newly diagnosed advanced ovarian carcinoma can influence the primary management choice for these patients. Gynecologic oncology 116: 483-488.

27. Aletti GD, Eisenhauer EL, Santillan A, Axtell A, Aletti G, et al. (2011) Identification of patient groups at highest risk from traditional approach to ovarian cancer treatment. Gynecologic oncology 120: 23-28.

28. Chi DS, Zivanovic O, Levinson KL, Kolev V, Huh J, et al. (2010) The incidence of major complications after the performance of extensive upper abdominal surgical procedures during primary cytoreduction of advanced ovarian, tubal, and peritoneal carcinomas. Gynecologic oncology 119: 38-42.

29. Kohl SK, Lewis SE, Tunnicliffe J, Lott RL, Spencer LT, et al. (2011) The College of American Pathologists and National Society for Histotechnology workload study. Archives of Pathology \& Laboratory Medicine 135: 728-736.

30. Aletti GD, Dowdy SC, Gostout BS, Jones MB, Stanhope RC, et al. (2009) Quality improvement in the surgical approach to advanced ovarian cancer: the Mayo Clinic experience. Journal of the American College of Surgeons 208 614-620.

31. Colgan TJ, Geldenhuys L (2012) The practice of pathology in Canada: decreasing pathologist supply and uncertain outcomes. Archives of pathology \& laboratory medicine 136: 90-94.

32. Neil A, Pfeffer S, Burnett L, BiPac (2013) Benchmarking in pathology: development of a benchmarking complexity unit and associated key performance indicators. Pathology 45: 66-70.

33. Burnett L, Wilson R, Pfeffer S, Lowry J, BiPac, et al. (2012) Benchmarking in pathology: development of an activity-based costing model. Pathology 44 644-653.

34. Valenstein PN, Souers R, Wilkinson DS, College of American P, et al. (2005) Staffing benchmarks for clinical laboratories: a College of American Pathologists $\mathrm{Q}$-probes study of staffing at 151 institutions. Archives of pathology \& laboratory medicine 129: 467-473.

35. Buesa RJ (2010) Productivity standards for histology laboratories. Annals of diagnostic pathology 14: 107-124.

36. Maung RT (2005) What is the best indicator to determine anatomic pathology workload? Canadian experience. American journal of clinical pathology 123: 45-55.

37. Tomaszewski JE, Abraham S, Bell K, Mourelatos Z, Reynolds C, et al. (1996) The measurement of complexity in surgical pathology. American journal of clinical pathology 106: S65-569.

38. Carr RA, Sanders DS, Stores OP, Smew FA, Parkes ME, et al. (2006) The Warwick system of prospective workload allocation in cellular pathology--an aid to subspecialisation: a comparison with the Royal College of Pathologists system. Journal of clinical pathology 59: 835-839. 